\title{
C-reactive protein and procalcitonin predict anastomotic leaks following colorectal cancer resections - a prospective study
}

\author{
Marek Zawadzki ${ }^{1}$, Roman Czarnecki ${ }^{1}$, Marek Rzaca ${ }^{1}$, Zbigniew Obuszko ${ }^{1}$, Vamsi Ramana Velchuru ${ }^{2}$, \\ Wojciech Witkiewicz ${ }^{1}$ \\ ${ }^{1}$ Department of Surgical Oncology, Research and Development Center, Regional Specialized Hospital, Wroclaw, Poland \\ ${ }^{2}$ Department of Colorectal Surgery, James Paget University Hospitals, Gorleston, UK
}

\begin{abstract}
Introduction: Early safe discharge is paramount for the success of ERAS following colorectal cancer resections. Anastomotic leakage (AL) has high morbidity, particularly if the patient has been discharged to the community. Aim: To evaluate whether C-reactive protein (CRP) and procalcitonin (PCT) can predict AL before early discharge. Material and methods: Fifty-five consecutive patients undergoing open and robotic colorectal cancer resections were included. C-reactive protein and PCT were measured pre-operatively, $8 \mathrm{~h}$ after incision, and on the first and third postoperative day. Thirty-day readmissions, re-operations and mortality were recorded.

Results: Twenty-nine patients underwent robotic and the remainder open $(n=26)$ resections. Five patients had AL. The mean CRP and PCT increased on postoperative day 1 (POD 1) and POD 3 in all patients. On POD 3, mean CRP was $114 \mathrm{mg} / \mathrm{l}$ in non-AL patients and $321 \mathrm{mg} / \mathrm{l}$ in AL patients $(p=0.0001)$. Mean $P C T$ on POD 3 was $0.56 \mathrm{ng} / \mathrm{ml}$ in the non-AL group and $10.4 \mathrm{ng} / \mathrm{ml}$ in AL patients $(p=0.017)$. On analysis of ROC and AUC curves, the cut-off for CRP on POD 3 was $245.64 \mathrm{mg} /$, with $100 \%$ sensitivity and 98\% specificity for AL. The cut-off for PCT on POD 3 was 3.83 $\mathrm{ng} / \mathrm{ml}$, with $75 \%$ sensitivity and $100 \%$ specificity for $\mathrm{AL}$.

Conclusions: C-reactive protein and PCT measurement on POD 3 following colorectal cancer resection can positively identify patients at low risk of anastomotic leakage.
\end{abstract}

Key words: colorectal cancer, robotic surgery, anastomosis leak, C-reactive protein, open surgery, colorectal.

\section{Introduction}

Anastomotic leakage (AL) is the most serious complication of colorectal resection, resulting in increased morbidity and mortality [1]. Incidence varies from $3 \%$ to $10 \%$ depending on patient characteristics and type of operation [2, 3]. Early diagnosis of AL is critical, but its clinical signs can be insidious. In most instances AL occurs around the $5^{\text {th }}-7^{\text {th }}$ postoperative day (POD), but it might develop any time from the day of index surgery to the third postoperative week $[2,4]$. With the standard postoperative protocols on POD 7 the patient is usually hospitalized and clinical symptoms herald the leakage.

In recent years, enhanced recovery after surgery (ERAS) has gained momentum in the management of the colorectal cancer patient. It reduces morbidity and shortens the hospital stay [5-7]. Early discharge benefits the patient and cuts medical care costs but carries a potential risk of developing AL when a patient is already out of the hospital. Delayed presentation and delayed diagnosis of AL can have devastating consequences. Any reliable marker to guide

\section{Address for correspondence}

Marek Zawadzki, Department of Research and Development Center, Regional Specialized Hospital, 73 a H. Kamińskiego St, 51-124 Wroclaw,

Poland, phone: +48 507046 930, e-mail: zawadzkimd@gmail.com 
safe early discharge can be a game changer in the present day practice of early discharges.

C-reactive protein (CRP) and procalcitonin (PCT) have been used as markers to identify sepsis in surgical departments. C-reactive protein levels on postoperative day 3-5 have been shown to provide valuable information with regards to the risk of $A L$ [8]. Procalcitonin is an another interesting marker of $A L$, but its value is less clear [9-11].

\section{Aim}

The aim of our study was to evaluate whether measurement of CRP and PCT on the $3^{\text {rd }}$ post-operative day predicts safe early discharge following robotic and open colorectal resection.

\section{Material and methods}

A prospective study involving 55 consecutive patients with colorectal cancer undergoing elective resection with anastomosis was performed between July 2013 and December 2014. The study was performed prior to the introduction of the ERAS protocol at our institution. Exclusions were patient less than 18 years, emergency surgery, R2 cancer resection, patients on immunosuppressive drugs and diabetics on high insulin doses. Patients were selected for a robotic approach based on the surgeon's discretion and the availability of the da Vinci system and trained staff. Institutional ethics committee approval was procured for the study. Detailed informed consent was obtained from all patients.

Demographics such as past medical history, tumour site and staging, perioperative outcomes and 30-day complications were prospectively collected. All patients were managed with a standard postoperative protocol with oral liquids on the first postoperative day, advancing to a liquid and solid diet on POD 2 and 3, respectively. Ambulation was encouraged on the day after surgery, and patients were discharged home when a normal diet was tolerated and bowel function had returned. Patients underwent additional postoperative diagnostic tests or treatment only in the event of symptoms or signs of a complication.

As per the agreed protocol, CRP and PCT were measured prior to surgery, $8 \mathrm{~h}$ after the incision, and on the first and third postoperative day $(0,+8 \mathrm{~h}$, POD 1, POD 3). Postoperative complications were recorded according to the Dindo and Clavien classifi- cation. Thirty-day admission rate, re-operations and mortality were recorded. All patients were seen and examined in an outpatient clinic within 30 days.

Procalcitonin was measured by the Vidas BRAHMS PCT automated test using the ELFA (enzyme-linked fluorescent assay) technique (BioMerieux SA, 69280 Marcy-l'Etoile, France). C-reactive protein was measured by the Multigent CRP Vario immunoturbidimetric test with the Architect $4100 \mathrm{Ci}$ analyzer (Abbott Diagnostics, California 95054, USA).

\section{Statistical analysis}

Continuous variables are reported as mean and standard deviation, whereas categorical variables are reported as number and percentage. Normality of data was assessed with the D'Agostino-Pearson test. The Friedman test was used for testing the changes in CRP and PCT levels at given time points. Nonparametric analysis was performed by the Mann-Whitney test to check differences between groups. The receiver operating characteristic (ROC) curve and the value of the area under the curve (AUC) were used to determine the diagnostic accuracy of CRP and PCT for detection of anastomotic leak.

\section{Results}

Fifty-five consecutive patients undergoing colorectal cancer resections were enrolled in the study. Patient characteristics and surgical data are shown in Table I. Twenty-nine patients underwent a robotic procedure and 26 patients had an open resection. There were 37 men and 18 women with an average age of 66.1 years and body mass index (BMI) of 27.4 $\mathrm{kg} / \mathrm{m}^{2}$. The robotic procedure took a significantly longer time than open resection (218 min vs. $133 \mathrm{~min}$, $p=0.0001$ ). The mean length of hospital stay including patients with anastomotic leakage was $6.7 \pm 3.2$ days. When excluding patients with $A L$ the mean length of hospital stay was $5.9 \pm 1.6$ days. There was no significant difference with regard to length of hospitalization between patients undergoing robotic and open procedures. There was no mortality. Five resections were complicated by anastomotic leak (9.1\%). Anastomotic leaks became clinically symptomatic between the $3^{\text {rd }}$ and $12^{\text {th }}$ postoperative day, and all required reoperation. Anastomotic leakage occurred exclusively among patients undergoing low anterior resection.

Difference in the CRP and PCT values with respect to postoperative course were observed within 
POD 0-3. (Table II, Figures 1 and 2) The mean value of CRP increased on POD 1 and POD 3 in all patients. The peak of CRP was significantly higher in the AL group only on POD 3. On the third postoperative day the mean values of CRP were $114 \mathrm{mg} / \mathrm{l}$ in non-AL patients and $321 \mathrm{mg} / \mathrm{L}$ in Al patients $(p=0.0001)$. Likewise, the mean PCT increased on POD 1 and 3 in all patients, but the rise of PCT was significantly higher among the patients with AL only on POD 3. The mean PCT on POD 1 was $2.0 \mathrm{ng} / \mathrm{ml}$ in non-AL and $3.8 \mathrm{ng} / \mathrm{ml}$ in AL patients ( $p=0.36$ ), whereas on POD 3 it was $0.56 \mathrm{ng} / \mathrm{ml}$ and $10.4 \mathrm{ng} / \mathrm{ml}$, respectively $(p=0.017)$.

Analysis of ROC curves showed that CRP on POD 3 had AUC of 0.996 and PCT on POD 3 had AUC of 0.85. A cut-off value for CRP on POD 3 was calculated at the level of $245.64 \mathrm{mg} / \mathrm{l}$, which resulted in $100 \%$ sensitivity and $98 \%$ specificity of AL. A cut-off value for PCT on POD 3 was calculated at the level of $3.83 \mathrm{ng} / \mathrm{ml}$ with $75 \%$ sensitivity and $100 \%$ specificity for AL (Table III, Figure 3 and 4).

\section{Discussion}

C-reactive protein and PCT have been used for many years for identifying septic complications. Our aim was to identify whether they can have a role in predicting anastomotic complications and help us to identify patients for safe early discharge according to the ERAS protocol. With the combination of a minimally invasive approach and ERAS protocol the average length of hospital stay could be safely reduced to four postoperative days [5]. Fast-track protocols encourage early patient discharge but might be associated with delayed diagnosis of anastomotic dehiscence. Early detection of AL is crucial for timely treatment, and delayed diagnosis is related to higher mortality. Ideal biochemical markers should have the potential to identify patients at risk of developing surgical complications prior to the occurrence of the first clinical symptoms. In theory this could facilitate distinction of patients amenable to safe discharge in the early postoperative period from those requiring closer monitoring and longer hospital stay.

C-reactive is a serum acute-phase reactant synthesized almost exclusively in the liver, released in response to stimulation by proinflammatory cytokines. Its production is part of a nonspecific acute-phase response to most forms of tissue damage, infection, inflammation, and malignant neoplasia. The synthesis of CRP increases rapidly, principally under the
Table I. Patients' $(n=55)$ demographics and perioperative data

\begin{tabular}{|c|c|}
\hline Parameter & Results \\
\hline Age, mean (SD) [years] & $66.1(11.2)$ \\
\hline \multicolumn{2}{|l|}{ Gender, $n(\%)$ : } \\
\hline Male & $37(67.3)$ \\
\hline Female & $18(32.7)$ \\
\hline \multicolumn{2}{|l|}{ ASA score, $n(\%)$ : } \\
\hline 1 & $11(20)$ \\
\hline 2 & $39(70.9)$ \\
\hline 3 & $5(9.1)$ \\
\hline BMI, mean (SD) $\left[\mathrm{kg} / \mathrm{m}^{2}\right]$ & $27.4(4.6)$ \\
\hline \multicolumn{2}{|l|}{ Pathology, T stage: } \\
\hline $\mathrm{T} 1$ & $4(7.4)$ \\
\hline $\mathrm{T} 2$ & $6(10.9)$ \\
\hline $\mathrm{T} 3$ & $36(65.4)$ \\
\hline $\mathrm{T} 4$ & $9(16.3)$ \\
\hline \multicolumn{2}{|l|}{ Type of operation, $n(\%)$ : } \\
\hline Right colectomy & $19(34.5)$ \\
\hline Left colectomy & $3(5.5)$ \\
\hline Sigmoid resection & $9(16.4)$ \\
\hline LAR & $24(43.6)$ \\
\hline \multicolumn{2}{|l|}{ Surgical technique, $n(\%)$ : } \\
\hline Robotic & $29(52.7)$ \\
\hline Open & $26(47.3)$ \\
\hline Operative time, mean (SD) [min] & $178(66.2)$ \\
\hline Length of hospital stay, mean (SD) [days] & $6.7(3.25)$ \\
\hline Anastomotic leak, $n$ (\%) & $5(9.1)$ \\
\hline
\end{tabular}

LAR - low anterior resection.

control of cytokines (interleukin-6) produced by macrophages and $T$ cells at the site of pathology. Subsequently, CRP binds macromolecular ligands on the surface of dead or dying cells as well as some types of bacteria and activates the complement system.

In healthy young adults, the median concentration of CRP is approximately $0.8 \mathrm{mg} / \mathrm{l}$, but, following an acute-phase stimulus, values may increase to more than $500 \mathrm{mg} / \mathrm{l}$. De novo synthesis in the liver starts rapidly after a single stimulus and reaches a peak within $48 \mathrm{~h}$. The plasma half-life of CRP is 
Table II. CRP and PCT values on POD 0-3 in non-AL and AL patients

\begin{tabular}{|c|c|c|c|c|c|c|c|}
\hline \multirow[t]{3}{*}{ Parameter } & \multicolumn{7}{|c|}{ Anastomotic leakage } \\
\hline & \multicolumn{3}{|c|}{ Non AL } & \multicolumn{3}{|c|}{$\mathrm{AL}$} & \multirow[t]{2}{*}{ Value of $p$} \\
\hline & Mean & SD & Median & Mean & SD & Median & \\
\hline CRP 0 & 13.995 & 27.4477 & 4.3 & 10.616 & 15.3131 & 1.69 & 0.76 \\
\hline$C R P+8 h$ & 13.179 & 22.0681 & 6.14 & 11.14 & 16.3107 & 4.4 & 0.74 \\
\hline CRP POD 1 & 110.004 & 34.2013 & 108.86 & 138.66 & 31.0772 & 133.5 & 0.082 \\
\hline CRP POD 3 & 114.398 & 59.5608 & 105.83 & 321.164 & 77.1356 & 299.61 & 0.0001 \\
\hline РСТ 0 & 0.133 & 0.4641 & 0.05 & 0.05 & 0 & 0.05 & 0.71 \\
\hline$P C T+8 h$ & 0.184 & 0.3149 & 0.07 & 0.066 & 0.02302 & 0.05 & 0.53 \\
\hline PCT POD 1 & 2.001 & 3.3283 & 0.72 & 3.826 & 4.2504 & 3.3 & 0.36 \\
\hline PCT POD 3 & 0.569 & 0.7358 & 0.22 & 10.475 & 14.5016 & 4.88 & 0.017 \\
\hline
\end{tabular}

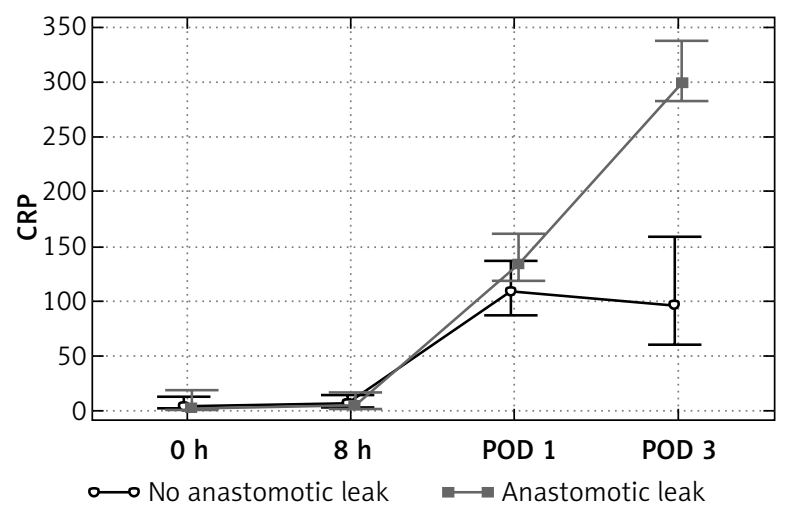

Figure 1. Evolution of median CRP over time

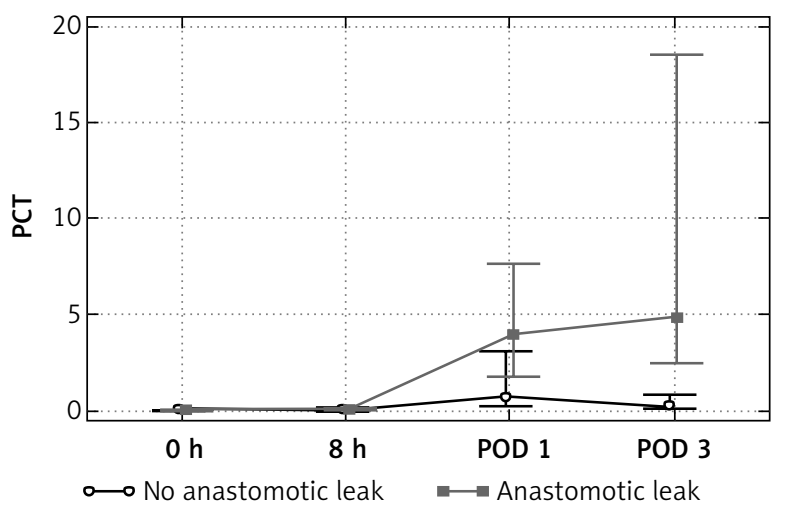

Figure 2. Evolution of median PCT over time

Table III. ROC curve analysis

\begin{tabular}{|lccccc|}
\hline Parameter & Sensitivity & Specificity & Cut-off & AUC & Value of $p$ \\
\hline CRP 0 & 60 & 68.75 & 1.69 & 0.546 & 0.79 \\
\hline CRP + 8 h & 40 & 83.72 & 1.69 & 0.549 & 0.77 \\
\hline CRP POD 1 & 80 & 72.34 & 125.3 & 0.74 & 0.043 \\
\hline CRP POD 3 & 100 & 97.87 & 245.64 & 0.996 & 0.0001 \\
\hline PCT 0 & 100 & 10.42 & 0.05 & 0.552 & 0.79 \\
\hline PCT + 8 h & 100 & 27.91 & 0.1 & 0.591 & 0.42 \\
\hline PCT POD 1 & 60 & 80.85 & 3.15 & 0.63 & 0.44 \\
\hline PCT POD 3 & 75 & 100 & 3.83 & 0.851 & 0.019 \\
\hline
\end{tabular}

about $19 \mathrm{~h}$ and is constant under all conditions of health and disease [12].

Following colorectal surgery CRP shows a rapid increase on POD 1 , reaching a peak on POD 2, fol- lowed by steady decline to a normal level thereafter. Patients with septic complications tend to have a higher peak of CRP on POD 3, which plateaus afterwards [13]. In 2012 Warschkow et al. published 
CRP

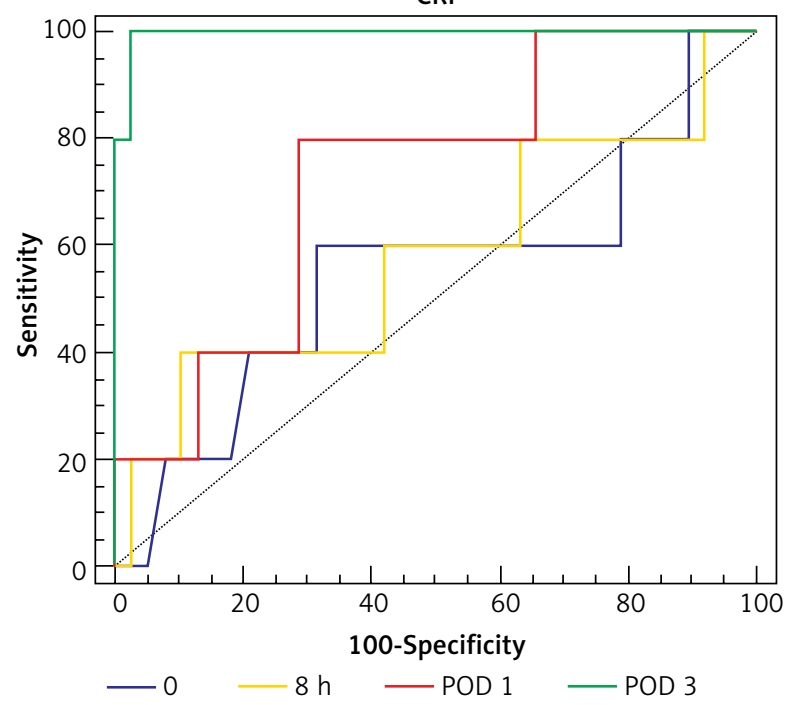

Figure 3. Receiver operating characteristic (ROC) curve for cut-off analysis of serum CRP in patients with $\mathrm{AL}$

a meta-analysis evaluating the predictive value of CRP for infectious complications after colorectal surgery [14]. They found that testing of CRP on POD 4 offers satisfactory accuracy of foreseeing AL and proposed a cut-off value of $135 \mathrm{mg} / \mathrm{l}$ with a negative predictive value of $89 \%$. More recently, Singh et al. published another meta-analysis $(n=2483)$ [8], with CRP as a leak predictor on POD 3, 4 and 5. The authors observed comparable diagnostic accuracy of CRP at the three abovementioned time points, with a high negative predictive value of $97 \%$ but a low positive predictive value of $21-23 \%$. These results confirmed that CRP is a useful negative test but not a good positive predictor of AL. In our study, CRP was significantly high on the 3rd post-operative day in the AL group, which might help with increasing suspicion of leak needing intense monitoring. In many instances, however, elevation of CRP might simply be due to another source of sepsis such as a wound or respiratory or urinary tract infection rather than anastomotic leak.

Procalcitonin is another promising plasma marker for identifying sepsis. Procalcitonin is a protein consisting of 116 amino acids, the peptide precursor of calcitonin, synthesized by the parafollicular C-cells of the thyroid gland. The release of PCT is induced specifically by bacterial endotoxins, and its levels do not increase following inflammation of non-infectious origin. In healthy individuals, serum

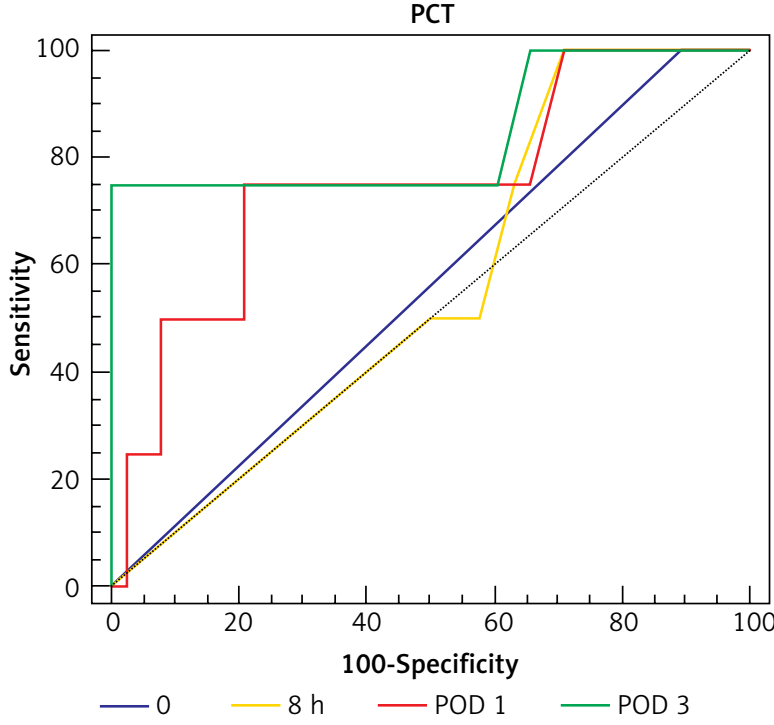

Figure 4. Receiver operating characteristic (ROC) curve for cut-off analysis of serum PCT in patients with $\mathrm{AL}$

concentrations of PCT are below $0.1 \mathrm{ng} / \mathrm{ml}$. In response to a bacterial infection, damage-associated molecular patterns (DAMPs) and pathogen-associated molecular patterns stimulate cells to produce PCT, which results in a significant increase in serum concentrations. Following surgery, on the first and second postoperative day PCT concentrations are commonly elevated in patients after major abdominal and vascular or thoracic operations and remain low among patients undergoing minor, aseptic procedures. It has been suggested that PCT is induced by a transient bacterial contamination or bacterial translocation during the operation or preparation of intestinal anastomoses. It has also been observed that patients with an abnormal postoperative course more frequently have increased PCT levels than patients with a normal postoperative course $[15,16]$.

Hence, PCT seems to be a more specific marker of septic complications than CRP. Lagoutte et al. first described kinetics of PCT after colorectal resection [9]. They evaluated a group of 100 patients, and in non-AL patients PCT levels rose on POD 1 to reach a peak on POD 2 and declined progressively. Patients with a leak had a higher peak of PCT on POD 1, followed by a decline afterwards. PCT showed the best accuracy of AL prognosis on POD 4, but its correlation with leakage was weaker than for CRP. In 2013, Garcia-Granero et al. analyzed CRP and PCT levels between POD 1 and 5 in 205 colorectal pa- 
tients with 11 major anastomotic leaks [10]. Procalcitonin was a satisfactory predictor of AL on POD 3, 4 and 5 (AUC >0.80), reaching its maximal value on the fifth postoperative day (AUC 0.867). Among analyzed variables (PCT, CRP, neutrophils on POD 3, 4, 5), PCT on POD 5 had the best accuracy in prognosis of anastomotic dehiscence. The authors proposed a PCT cut-off value of $0.31 \mathrm{ng} / \mathrm{ml}$ on POD 5. Similar results were recently obtained by Giaccaglia et al. [11] In our study, if PCT was significantly higher on the third day, an anastomotic leak should be suspected, requiring intense monitoring.

Our study is intended to aid decision making for early discharge following colorectal cancer resections, particularly in the age of ERAS protocols. We found CRP and PCT levels on POD 3 to be significantly higher in patients with AL. The specificity and sensitivity of both markers were similar and ROC analysis suggested higher accuracy of CRP. The present study confirmed that CRP and PCT are reliable early predictors of anastomotic dehiscence. We believe that these findings together with previous results support routine implementation of CRP and PCT testing on POD 3 in fast-track protocols.

At least 2 issues require further investigation. In accordance with previous reports we found no advantage of PCT over CRP in prediction of AL. In the global tendency of reducing health care expenses the cost of additional testing must be taken into consideration. The cost of the PCT test is approximately 8 times higher than the cost of the CRP test ( $€ 21$ vs. $€ 2.50$ ). Due to the low cost of the test, it might be advisable to recommend CRP as a routine test on POD 3 and use PCT as a second line test, verifying the abnormal CRP results. Secondly, an additional investigation is necessary to determine whether such routine testing on POD 3 should be recommended to all restorative colorectal resections or selectively to patients at higher risk of anastomotic leakage (left sided or rectal resections, elderly patients, obese individuals, etc.)

Most previous studies evaluated CRP and PCT in patients undergoing open procedures. In a study involving patients undergoing laparoscopic and open colonic resection, laparoscopic surgery was shown to have attenuated stress responses and improved preservation of immune function [17]. If the biokinetics of plasma markers following laparoscopy differs from open surgery, future studies need to focus more on a cohort of patients undergoing a minimally invasive approach.
It is paramount to understand that low CRP and PCT had satisfactory negative predictive value to rule out septic complications and confirm safe discharge from the hospital. Nevertheless, the decision for re-intervention is based on positive predictive value, which is low for both CRP and PCT. The verdict of reoperation is often difficult and based mostly on clinical judgment and probably by imaging. Additional tests are non-specific and frequently uninformative. Lately, newer markers such as calprotectin, fatty acid binding protein or peritoneal fluid cytokines have been investigated, with promising results $[18,19]$.

The small sample size and only five cases of anastomotic dehiscence are obvious limitations of the present study. The evidence from the literature suggests a cut-off value of $172 \mathrm{mg} / \mathrm{l}$ for CRP and $0.64 \mathrm{ng} / \mathrm{ml}$ for PCT on POD $3[8,10]$. In our study the calculated cut-off value on POD 3 was $245 \mathrm{mg} / \mathrm{l}$ for CRP and $3.83 \mathrm{ng} / \mathrm{ml}$ for PCT. This difference is probably an effect of the small sample size of the study. The fact that more than half of the patients underwent a robotic procedure might be another factor influencing our results.

\section{Conclusions}

A single measurement of C-reactive protein and procalcitonin on the third postoperative day following colorectal cancer resection allows identification of patients at low risk of anastomotic leakage. Both plasma markers can be used routinely to guide safe discharge of patients treated with the ERAS protocol and reduce the hazard of delayed diagnosis of anastomotic dehiscence. Further research for early, more specific predictors of anastomotic leakage is advocated.

\section{Conflict of interest}

The authors declare no conflict of interest.

\section{Acknowledgments}

This publication is part of Project „WroVasc - Integrated Cardiovascular Centre", co-financed by the European Regional Development Fund, within Innovative Economy Operational Program, 2007-2013 realized in Regional Specialist Hospital, Research and Development Center in Wroclaw. "European Funds for the development of innovative economy".

The publication was funded by the Foundation of Medical Innovations WroVasc. 


\section{References}

1. Bakker IS, Grossmann I, Henneman D, et al. Risk factors for anastomotic leakage and leak-related mortality after colonic cancer surgery in a nationwide audit. Br J Surg 2014; 101: 424-32.

2. Krarup PM, Jorgensen LN, Andreasen AH, Harling H. A nationwide study on anastomotic leakage after colonic cancer surgery. Colorectal Dis 2012; 14: 661-7.

3. Kube R, Mroczkowski P, Granowski D, et al. Anastomotic leakage after colon cancer surgery: a predictor of significant morbidity and hospital mortality, and diminished tumour-free survival. Eur J Surg Oncol 2010; 36: 120-4.

4. Blumetti J, Chaudhry V, Cintron JR, et al. Management of anastomotic leak: lessons learned from a large colon and rectal surgery training program. World J Surg 2014; 38: 985-91.

5. Greco M, Capretti G, Beretta L, et al. Enhanced recovery program in colorectal surgery: a meta-analysis of randomized controlled trials. World I Surg 2014; 38: 1531-41.

6. Haverkamp MP, de Roos MAJ, Ong KH. The ERAS protocol reduces the length of stay after laparoscopic colectomies. Surg Endosc 2012; 2 6: 361-7.

7. Kisialeuski M, Pędziwiatr M, Matłok M, et al. Enhanced recovery after colorectal surgery in elderly patients. Videosurgery Miniinv 2015; 10: 30-6.

8. Singh, PP, Zeng ISL, Srinivasa S, et al. Systematic review and meta-analysis of use of serum C-reactive protein levels to predict anastomotic leak after colorectal surgery. Br J Surg 2014; 101: 339-46.

9. Lagoutte N, Facy O, Ravoire A, et al. C-reactive protein and procalcitonin for the early detection of anastomotic leakage after elective colorectal surgery: pilot study in 100 patients. J Visc Surg 2012; 149: e345-9.

10. Garcia-Granero A, Frasson M, Flor-Lorente B, et al. Procalcitonin and C-reactive protein as early predictors of anastomotic leak in colorectal surgery: a prospective observational study. Dis CoIon Rectum 2013; 56: 475-83.

11. Giaccaglia V, Salvi PF, Cunsolo GV, et al. Procalcitonin, as an early biomarker of colorectal anastomotic leak, facilitates enhanced recovery after surgery. J Crit Care 2014; 29: 528-32.

12. Pepys MB, Hirschfield GM. C-reactive protein: a critical update. J Clin Invest 2003; 111: 1805-12.

13. MacKay GJ, Molloy RG, O'Dwyer PJ. C-reactive protein as a predictor of postoperative infective complications following elective colorectal resection. Colorectal Dis 2011; 13: 583-7.

14. Warschkow R, Beutner U, Steffen T, et al. Safe and early discharge after colorectal surgery due to C-reactive protein: a diagnostic meta-analysis of 1832 patients. Ann Surg 2012; 256: 245-50.

15. Biron BM, Ayala A, Lomas-Neira JL. Biomarkers for sepsis: what is and what might be? Biomark Insights 2015; 10 (Suppl. 4): 7-17.

16. Meisner M, Tschaikowsky K, Hutzler A, et al. Postoperative plasma concentrations of procalcitonin after different types of surgery. Intensive Care Med 1998; 24: 680-4.

17. Veenhof AA, Vlug MS, van der Pas MH, et al. Surgical stress response and postoperative immune function after laparoscopy or open surgery with fast track or standard perioperative care: a randomized trial. Ann Surg 2012; 255: 216-21.
18. Reisinger KW, Poeze M, Hulsewé KW, et al. Accurate prediction of anastomotic leakage after colorectal surgery using plasma markers for intestinal damage and inflammation. J Am Coll Surg 2014; 219: 744-51.

19. Cini C, Wolthuis A, D'Hoore A. Peritoneal fluid cytokines and matrix metalloproteinases as early markers of anastomotic leakage in colorectal anastomosis: a literature review and meta-analysis. Colorectal Dis 2013; 15: 1070-7.

Received: 22.07.2015, accepted: 20.10.2015. 